Revue Revue de l'histoire des religions

de Ihistoire des religions

Théologie politique et sciences sociales : autour d'Erik Peterson (1890-1960)

\title{
David GILBERT, « Le Grand secret de la vocation ». Louis
} Tronson (1622-1700)

Paris, Honoré Champion («Bibliothèque d'études des mondes chrétiens », 12), 2018

\section{Bruno Restif}

\section{OpenEdition}

\section{Journals}

Édition électronique

URL : https://journals.openedition.org/rhr/11410

DOI : 10.4000/rhr.11410

ISSN : 2105-2573

Éditeur

Armand Colin

Édition imprimée

Date de publication : 1 septembre 2021

Pagination : 581-584

ISBN : 978-2-200-93377-7

ISSN : 0035-1423

Référence électronique

Bruno Restif, « David GILBERT, «Le Grand secret de la vocation ». Louis Tronson (1622-1700) », Revue de I'histoire des religions [En ligne], 3 | 2021, mis en ligne le 01 septembre 2021, consulté le 04 octobre 2021. URL : http://journals.openedition.org/rhr/11410 ; DOI : https://doi.org/10.4000/rhr.11410

Ce document a été généré automatiquement le 4 octobre 2021.

Tous droits réservés 


\section{David GILBERT, « Le Grand secret de la vocation ». Louis Tronson (1622-1700)}

Paris, Honoré Champion («Bibliothèque d'études des mondes

chrétiens ", 12), 2018

\section{Bruno Restif}

\section{RÉFÉRENCE}

David GILBERT, « Le Grand secret de la vocation ». Louis Tronson (1622-1700), Paris, Honoré Champion («Bibliothèque d'études des mondes chrétiens »,12), 2018, 565 p., 23,5 cm, $68 €$, ISBN 978-2-7453-4716-9.

1 Cet ouvrage reprend le texte d'une thèse soutenue en 2015 en vue de l'obtention d'un doctorat conjoint en théologie et en histoire. Le refus d'une séparation trop nette entre ces deux disciplines peut être loué, mais il est évident que leur articulation pose des problèmes épistémologiques complexes dont il n'est pas possible de faire l'économie. La prise en compte de ces enjeux permet des investigations nouvelles aux résultats stimulants, comme l'a montré Giorgio Agamben, mais ici la démarche reste classique, la réflexion sur l'aspect transdisciplinaire de l'entreprise est peu approfondie, et cela explique que l'histoire y apparaisse, malgré des dénégations, comme ancilla theologiae. Le projet lui-même est théologique, ce que masque un titre un peu trompeur. En effet, ce livre traite non pas d'un seul sujet, qui serait celui de la vocation chez Louis Tronson, mais de deux: la question de la vocation ecclésiastique en milieu catholique du $\mathrm{XVI}^{\mathrm{e}}$ siècle à nos jours, et l'apport jugé essentiel sur ce sujet de Louis Tronson. Et c'est parce qu'il y a un effort réel d'insertion de ce personnage, dont ne traitent ici que certains chapitres, dans son milieu, que l'entreprise est présentée comme historienne, mais cette contextualisation des propos de Tronson est suivie de leur décontextualisation, à travers leur relecture vers $1900 \ldots$ ou aujourd'hui.

2 Pour ces raisons, ce livre peut dérouter l'historien qui s'attendrait à une étude portant spécifiquement sur la vie, l'œuvre et la pensée de celui qui fut directeur du séminaire 
de Saint-Sulpice à partir de 1657, supérieur général de la Compagnie de Saint-Sulpice à partir de 1676, arbitre dans la querelle du quiétisme, et acteur tardif à l'esprit pratique de ce que Bremond appelait l'École française de spiritualité. L'aspect historien de l'entreprise de David Gilbert se traduit surtout par une présentation détaillée, et donc utile, des sources, manuscrites et imprimées, par de nombreuses citations - mais l'orthographe est volontairement modifiée, si bien qu'il faut retourner aux sources pour réutiliser les citations -, et par le recours à une très classique histoire politique, sociale et religieuse. Émergent comme références les noms de Roland Mousnier, Jean de Viguerie et Dominique Dinet, notamment, et l'on peut penser qu'ils ont été privilégiés parce qu'ils présentaient un profil à peu près commun, plus assimilable idéologiquement que celui de chercheurs plus récents ou plus originaux. Naturellement, D. Gilbert a bien le droit de choisir un positionnement, qui ne saurait poser problème qu'à partir du moment où il pèse sur la qualité de l'argumentation. Mais justement, c'est là que le bât blesse de la façon la plus nette. L'emploi répété du mot «sociologie " peine à masquer la faiblesse d'une mise en œuvre de ce type, alors que l'école de sociographie religieuse française est connue pour avoir produit de nombreuses études qui peuvent être utilisées pour traiter de la vocation ecclésiastique. Ce qui est vrai de la discipline historique française l'est aussi des travaux allemands et suisses, si bien que la pensée luthérienne est présentée ici hors-sol, sans la moindre référence à la réalité des Églises princières et de la confessionnalisation, et l'on peut dire la même chose de la conception calviniste, qui n'est pas référée de façon suffisamment nette au mode de fonctionnement presbytéro-synodal. L'apport des sociologues à l'histoire manque lui aussi à une véritable saisie du terrain, qu'il s'agisse des travaux de Norbert Elias à propos des significations de l'habit et de la civilité, ou de ceux de Pierre Bourdieu dès lors que D. Gilbert compare magistrature et clergé, service de l'État et service de l'Église. Car l'auteur traite de nombreux sujets passionnants, susceptibles de mieux éclairer un objet difficile à cerner, ce qui rend d'autant plus regrettable l'absence de recours à des outils disciplinaires qui sont aujourd'hui bien connus. Sans surprise, c'est dans le domaine de l'histoire ecclésiastique traditionnelle, inspirée à la fois par le modèle de l'histoire politique et par celui de l'histoire spirituelle à la Bremond, que la partie historienne de l'ouvrage présente une réussite. En ce domaine, D. Gilbert ne s'intéresse pas seulement à Tronson, mais également à Bérulle, Olier et Saint-Cyran, s'employant à montrer leurs points communs et leurs différences de positionnement, ainsi qu'à pister les influences des uns sur les autres, avec un résultat fragile sur la question, pourtant essentielle dans l'argumentation de D. Gilbert, d'une influence complexe de Saint-Cyran sur la pensée de Tronson.

3 Théologique, cette entreprise procède par analyse de buttes-témoins, sortes de loci theologici qui ne se confondent pas avec des notions mais avec des auteurs ou des querelles: Clichtove, Luther et Calvin, puis Bérulle, Saint-Cyran et Tronson, enfin la querelle entre Louis Branchereau et Joseph Lahitton vers 1900, des appréciations très personnelles de D. Gilbert rappelant que l'essentiel est de savoir ce qu'il est possible d'en tirer aujourd'hui. De nombreuses analyses s'appuient largement sur des travaux déjà existants, l'objectif du livre étant de montrer que Tronson, qui n'est pas un personnage haut en couleur et qui est demeuré jusqu'ici insuffisamment étudié, a beaucoup à nous dire, si bien que l'apport le plus original de D. Gilbert porte bien sur ce personnage et sa pensée. Mais cette méthode de travail consistant à ne s'intéresser qu'à des buttes-témoins, qui est proche de l'histoire des idées à l'ancienne, pose au moins deux problèmes. Le premier est celui du choix : pourquoi n'avoir pas choisi Ignace de 
Loyola et sa pratique du discernement? Pourquoi avoir évacué à bon compte les séminaires de la seconde moitié du $\mathrm{XVI}^{\mathrm{e}}$ siècle, dont la réussite est peu spectaculaire mais qui étaient porteurs d'un projet, et les conciles provinciaux de la même période ? Le second problème est celui du saut entre les buttes : on peine à croire que Bérulle et Saint-Cyran aient été inspirés par Calvin plutôt que par les évolutions post-tridentines.

L'auteur a raison lorsqu'il affirme que la question de la vocation se pose de façon nouvelle dès lors qu'il y a éclatement de la Chrétienté au xvi siècle, mais ce n'est évidemment pas parce qu'il y aurait une revendication d'un exercice du libre arbitre contre l'institution, de la part de Luther et de Calvin. On peut regretter à ce sujet que la présentation de la pensée calvinienne soit faite sans référence suffisamment explicite à la prédestination, qui est ici un enjeu majeur. D. Gilbert montre, peut-être un peu trop à travers les lunettes de Lahitton et Branchereau, que s'affrontent sur la longue durée des partisans de la vocation extérieure, décision de l'Église qui envoie en mission, et des partisans de la vocation intérieure, plus subjective en apparence, qui pourrait se traduire dans l'inclination ou l'attrait d'un individu pour le ministère ecclésiastique. On est convaincu sans peine que des positions médianes peuvent être prises en compte.

La vocation est certes un appel, comme l'indique son étymologie, mais la seule vocation objective est l'appel de Dieu, qu'il est logique de vouloir identifier de la façon la moins incertaine possible, comme le fait Saint-Cyran. La vocation intérieure peut donc n'avoir aucun rapport avec l'attrait pour raisons mondaines, qui, lui, peut fort bien se combiner avec un appel de l'Église institutionnelle au temps du système bénéficial... ou en d'autres temps. Il y a donc des enjeux ecclésiologiques considérables, et les scrupules de Saint-Cyran n'ont rien d'étrange, même s'ils sont potentiellement déstabilisateurs. On peut penser qu'il y a en partie une opposition entre sensibilité thomiste, qui insiste sur l'efficacité objective du sacrement, et sensibilité augustinienne, très attentive à l'intériorité, ce qui contribuerait, avec la question de la prédestination, à expliquer la possibilité d'un rapprochement entre les conceptions de Saint-Cyran et de Calvin. En revanche, l'opposition entre " état » et «fonctions », sur laquelle insiste D. Gilbert dans la lignée du néothomiste Jacques Maritain, sous-estime la puissance, certes progressivement déclinante, du dionysisme, qui annihile toute possibilité d'opposition entre ces deux termes, dans la France du XvII siècle. Tronson, en conciliateur parfois besogneux, tente de combiner tous les critères qui peuvent être objectivés : idonéité, rectitude d'intention, acceptation par l'évêque, "sainteté ", "dégoût du monde ", "dextérité pour les fonctions ecclésiastiques », etc., les listes variant suivant les écrits. D. Gilbert nous convainc de l'apport de Tronson, et écrit des pages passionnantes sur l'identification des marques de la vocation intérieure que l'Église peut prendre en compte pour confier la mission du ministère.

Enfin, si l'on se place sur le terrain de D. Gilbert, on peut exprimer le souhait que Tronson et Saint-Cyran soient aujourd'hui lus par les séminaristes, mais aussi par les laïcs (auto)promus dans l'Église à des fonctions qui ne sont pas purement matérielles et qui doivent donc poser les mêmes questions. 


\section{AUTEURS}

BRUNO RESTIF

Université de Reims Champagne-Ardenne. 